\title{
Great-Expectations The Strength of Canadian Bridge Infrastructure and the threat of Climate Change
}

\author{
Lamya Amleh \\ Faculty of Engineering and Architectural Science, Ryerson University \\ Toronto, Ontario, Canada
}

Climate change is possibly the greatest challenge of our time and a credible existential threat to society as we know it. Transportation infrastructure constitute the essential veins and arteries that allow for the flow of energy, water, and essential goods to and from our cities and communities. Like the natural world, this built environment is vulnerable to the effects of climate change. These effects are already visible across the globe, and have been growing progressively worse over the last 50 years.

The deterioration rates of built infrastructure are a subject of serious concern within the engineering community; climate change is predicted to accelerate these rates and to increase the frequency and intensity of extreme weather effects. These changes will have considerable effects on built infrastructure, introducing increased environmental stresses, causing higher physical loads associated with precipitation as well as hydrostatic and hydrodynamic loads due to flooding.

One of the central challenges to the engineering community, and more generally to those developing climate-change adaptation strategies is the inherent uncertainty in future climate models; though there is wide consensus that the climate is changing, reliable predictions of how those changes manifest are still difficult to make. In response, the engineer concerned with the design of future infrastructure has a vested interest in the investment and further development of advances in climate science. However, in the absence of new breakthroughs and better predictions, probabilistic risk analysis becomes an essential tool in the bridge and transportation infrastructure design process.

This paper presents a comprehensive review of literatures found on bridge infrastructure adaptation to climate change. The paper starts with a global perspective on climate change followed by the Canadian perspective describing the impacts of climate change on transportation agencies activities and actions departments are taking to adapt. The paper concludes with a discussion of immediate risks and longer-term vulnerabilities, information gaps, and other challenges. 